\title{
Avaliação do risco potencial: da teoria à prática em Vigilância Sanitária
}

\section{Potential risk assessment: from theory to practice in Health Surveillance}

\author{
Marcus Vinícius Teixeira \\ Navarro,** iD \\ Eliana Auxiliadora Magalhães \\ Costa" ID \\ Luciana Freitas"II \\ Vanessa Lorena Sousa de \\ Medeiros Freitas ${ }^{\mathrm{IV}}$ (iD \\ Carina Kindermann $\sqrt{ }$ \\ Luiz Gustavo da Cruz Duarte'
}

Instituto Federal da Bahia, Salvador, BA, Brasil

" Universidade do Estado da Bahia, Salvador, BA, Brasil

III Vigilância Municipal de Salvador, Salvador, BA, Brasil

iv Subcoordenadoria de Vigilância Sanitária do Rio Grande do Norte, Natal, RN, Brasil

$\checkmark$ Diretoria de Vigilância Sanitária do Estado de Santa Catarina, Florianópolis, SC, Brasil

\section{* E-mail: navarro@ifba.edu.br}

\begin{abstract}
RESUMO
Introdução: 0 grande desafio do controle sanitário é avaliar o risco potencial de seus múltiplos objetos de ação, utilizando os resultados para selecionar as melhores estratégias, tarefa difícil de ser operacionalizada no cotidiano da Vigilância Sanitária (Visa). Objetivo: Apresentar a operacionalização do Modelo de Avaliação de Riscos Potenciais (MARP) em serviços de saúde do estado de Santa Catarina, de modo a subsidiar reflexões acerca da aplicabilidade desse método. Método: Estudo descritivo de casos múltiplos holísticos. A unidade de análise foi o controle sanitário de serviços de saúde realizado pela Visa de Santa Catarina, na Grande Florianópolis, tendo sido estudados $15(42,9 \%)$ dos 35 hospitais dessa região. A estratégia de avaliação de risco foi o MARP com aplicação de Roteiros Objetivos de Inspeção, nos Centros Cirúrgicos (CC), nas Centrais de Material Esterelizado (CME) e nas Unidades de Terapia Intensiva (UTI), nos anos 2017 a 2019. Resultados: As UTI apresentaram os maiores percentuais de risco aceitável e os menores de risco não aceitável, enquanto as CME apresentaram os menores percentuais de risco aceitável e os maiores de risco não aceitável. Os percentuais de risco potencial aceitável são maiores nos CC dos hospitais de grande porte, nas UTI dos hospitais de médio porte e ausente nas CME e nos CC dos hospitais de pequeno porte. Conclusões: 0 MARP favorece o gerenciamento dos riscos em Visa e o direcionamento de suas ações de controle sanitário. Possui limitações, ao necessitar de um sistema de informação e requer compreensão dos resultados dos dados, tendo em vista que risco e benefício são conceitos desafiadores e devem ser analisados num contexto definido.
\end{abstract}

PALAVRAS-CHAVE: Vigilância Sanitária; Risco; Regulação; Serviços de Saúde

\section{ABSTRACT}

Introduction: The great challenge of health control is to evaluate the potential risk of its multiple objects of action, using the results to select the best strategies, a task difficult to be operationalized in the daily routine of the Sanitary Surveillance (Visa). Objective: To present the operationalization of the Model of Potential Risk Assessment (MARP) in health services in the state of Santa Catarina (SC), in order to support reflections about the applicability of this method. Method: Descriptive study of holistic multiple cases. The unit of analysis was the sanitary control of health services carried out by the Visa of SC, in Greater Florianópolis, and $15(42.9 \%)$ of the 35 hospitals in this region. The risk assessment strategy was MARP with application of Objective Inspection Scripts, in the Surgical Center (SC), Sterilized Material Center (SMC) and Intensive Care Unit (ICU), in the years 2017 to 2019. Results: The ICU presented the highest percentages of acceptable risk and the lowest percentages of unacceptable risk, while the CME presented the lowest acceptable risk percentages and the highest percentages of unacceptable risk. The percentages of acceptable potential risk are higher in the CC of large hospitals, in the ICU of medium-sized hospitals and absent in the CME and CC of small hospitals. Conclusions: MARP favors the management of risks in Visa and the direction of its health control actions. It has limitations, when requiring an information system and needs understanding of the results of the data, considering that risk and benefit are challenging concepts and should be analyzed in a defined context.

KEYWORDS: Health Surveillance; Risk; Regulation; Health Services 


\section{INTRODUÇ̃̃O}

A Vigilância Sanitária (Visa) é parte constituinte do Sistema Único de Saúde (SUS), integra a saúde coletiva enquanto campo de interesse teórico e prático e tem a responsabilidade constitucional de prevenir e controlar riscos relacionados a produtos, processos e serviços de interesse da saúde humana'.

Os referenciais do risco são utilizados em várias áreas do conhecimento e na saúde têm se consolidado como "probabilidade de ocorrência de um evento adverso e sua severidade, usado como medida de impacto ou associado a diferenciais de morbidade ou mortalidade entre grupos, com e sem um determinado atributo, constituindo-se no risco epidemiológico ou clássico"2, adquirindo, então, uma percepção quantitativa, baseada na associabilidade de eventos para inferir probabilidades de suscetibilidade segundo o grau de exposição a determinados agentes ${ }^{3,4,5}$.

Independentemente da polissemia do termo, o risco é elemento central nas ações de Visa e deve ser o indutor de tomada de decisão racional. Entretanto, para a Visa, além do conceito de risco epidemiológico sustentado por evidência científica probabilística, faz-se necessário, um conceito que ultrapasse a relação causa-efeito, tão típica e evidenciada do conceito de risco clássico, para um entendimento de risco que possa acontecer mesmo na ausência desse nexo causal ${ }^{3,6}$.

Estamos falando do risco potencial, como possibilidade do dano, uma vez que a Visa, na prática do controle sanitário de seus múltiplos, distintos e crescentes objetos de ação (água e alimentos, resíduos, cosméticos, germicidas, medicamentos, sangue, serviços de saúde, radiologia, escolas, comida de rua, presídios, cemitérios etc.), na grande maioria das situações, não consegue estabelecer a associação de causa e efeito comum dos estudos e da vigilância epidemiológica ${ }^{3,6}$.

Sabe-se que os objetos de atuação da Visa têm características particulares: são de interesse da saúde humana; produzem benefícios e possuem riscos intrínsecos. Assim, caso a relação risco versus benefício seja favorável ao benefício, é imprescindível o entendimento da aceitabilidade do risco em determinados contextos do controle sanitário, processo esse que não depende apenas de avaliações técnico-científicas, mas também da percepção do risco associado a aspectos culturais e ao uso do benefício ${ }^{3,5,6}$.

Benefício é um conceito em construção na área da saúde e vem sendo utilizado por órgãos reguladores nas áreas de controle de medicamentos, alimentos e dispositivos médicos. Pode ser compreendido como o oposto do risco e simetricamente entendido como a condição ou o contexto de exposição a um determinado atributo de uma tecnologia, que possui determinada probabilidade de causar situação favorável, com determinada magnitude. São os "efeitos favoráveis" para uma população-alvo associados com um processo, serviço ou produto $0^{5,7,8,9,10}$.

Assim, enquanto o risco potencial pode ser entendido como a possibilidade de ocorrência de um efeito desfavorável, sem necessariamente descrever o efeito e sua probabilidade, o benefício potencial pode ser definido como a possibilidade de ocorrência de um efeito favorável, sem necessariamente descrever o efeito e sua probabilidade ${ }^{7,8,910}$.

Os conceitos e a avaliação de risco e benefício são desafiadores quando da aplicabilidade desses na prática do controle sanitário realizado pelas Visa, sendo centrais para a tomada de decisão regulatória, contribuindo para ações sanitárias eficazes, transparentes e de confiança entre os setores envolvidos ${ }^{3,4,9}$.

Esses conceitos subsidiam a avaliação da prática diária do controle da Visa, a exemplo de como determinar o risco de um paciente cirúrgico submetido a um procedimento com instrumental sem controle de esterilização. Auxilia também na classificação do risco relacionado à ausência de protocolos assistenciais padrão, dentre outras inúmeras situações cotidianas e emblemáticas para o profissional da Visa que, muitas vezes, movido por racionalidades do senso comum, realiza intervenções sem efetividade, de alto custo econômico e ambiental, e que em nada contribuem para o controle dos riscos e benefícios em serviços de saúde ${ }^{11}$.

Adicionalmente, para o controle sanitário de serviços de saúde, as Visa utilizam roteiros de inspeção, que constituem em instrumentos de avaliação com respostas binárias e que classificam as situações identificadas nos serviços em "conformes ou não conformes", "sim ou não", "risco alto, médio ou baixo", com considerável parcela de subjetividade, tanto em relação ao serviço, produto ou processo a ser avaliado, quanto as variadas interpretações dos marcos regulatórios utilizados para esse fim $^{3,11}$.

Neste sentido, o grande desafio do controle sanitário é avaliar o risco e a garantia do benefício de seus múltiplos objetos de ação sem subestimá-los e sem supervalorizá-los, bem como utilizar os dados da avaliação de risco e benefício para implementar o seu gerenciamento, selecionando as melhores políticas e estratégias para o controle do risco, tarefa difícil de ser operacionalizada no cotidiano da Visa.

Modelo de Avaliação de Risco Potenciais e Roteiro Objetivo de Inspeção (MARP \& ROI)

Para operacionalização do conceito de risco potencial (RP), Navarro ${ }^{12}$ desenvolveu o Modelo de Avaliação de Riscos Potenciais (MARP), apresentando uma metodologia qualitativa e quantitativa na qual, por meio da classificação dos indicadores de controle de riscos, que são itens retirados das normas sanitárias e que compõem um instrumento de inspeção, é possível mensurar o risco potencial do serviço avaliado.

Como forma de tornar a avaliação mais realista possível, o MARP quantifica os indicadores na seguinte escala: 0 (a pior situação, em que há ausência ou descumprimento total das normas); 1: sofrível (atende menos do que parcialmente a norma); 2: razoável (atende a norma, mas não integralmente); 3: bom 
(atende ao que a norma determina); 4: ótimo (atende mais do que a norma determina); 5: excelente (apresenta requisitos de excelência de qualidade assistencial); NA: não avaliado ${ }^{12}$.

Os indicadores utilizados no instrumento de inspeção são classificados em críticos $\left(I_{C}\right)$ e não críticos $\left(I_{N C}\right)$, a depender da gravidade e do possível risco associado. Os críticos podem levar o sistema ao risco potencial máximo caso estejam avaliados como a situação zero. Os não críticos influenciam no valor do risco, mas não determinam o risco potencial máximo, exceto quando o conjunto inteiro destes for avaliado na situação zero ${ }^{12}$.

Assim, o RP foi definido como:

$$
R_{P}=\sqrt{\sqrt[N]{\prod_{i=1}^{N} I_{C_{i}} x \frac{\sum_{j=1}^{M} I_{N C_{j}}}{M}}}
$$

Sendo o primeiro termo da raiz quadrada a média geométrica dos $\mathrm{N}$ indicadores críticos $\left(\mathrm{Ic}_{\mathrm{i}}\right)$ e o segundo termo a média aritmética dos $M$ indicadores não críticos $\left(I_{N C_{j}}\right)$.

Entretanto, a classificação dos indicadores entre zero e cinco continuou trazendo muita subjetividade ao MARP. Visando aprimorar o método incorporando transparência e objetividade, foi desenvolvida uma nova tecnologia de inspeção denominada Roteiro Objetivo de Inspeção (ROI) que descreve itens de verificação das unidades de avaliação, trazendo maior detalhamento das possíveis situações encontradas, como exemplificado na Figura 1. Desse modo, o ROI possibilita a reprodutibilidade da avaliação de um serviço independente do técnico que faça uso desse instrumento ${ }^{6}$.

O ROI pode ser construído para as mais diversas áreas de Visa e no estado de Santa Catarina (SC) já foi elaborado para os seguintes serviços: Unidade de Terapia Intensiva (UTI - neonatal, pediátrica e adulto), Centro Cirúrgico (CC), urgência e emergência, endoscopia (tipos I, II e III), Unidade de Internação, Posto de Coleta Laboratorial, farmácias (hospitalar, manipulação e sem manipulação), laboratórios (microbiologia, clínico, anatomia patológica e analítico), segurança do paciente, Central de Material e Esterilização (CME - tipos I e II), diálise, nutrição e dietética, serviços de radiologia médica, medicina nuclear e radioterapia.

Os resultados das avaliações classificam as unidades inspecionadas em três níveis de risco: aceitável, tolerável e inaceitável; a depender do valor de risco potencial calculado em cada inspeção, conforme o Quadro.

A classificação do valor de RP sinaliza possíveis tomadas de decisão. Se o RP é alto ( $R P>0,360)$, a tendência é que venha a ocorrer interdição ou suspensão de atividades no serviço. Quando o resultado é tolerável, o serviço pode receber notificação para correção das não conformidades em prazo definido, e se o risco é aceitável, certamente haverá a liberação da licença sanitária.

Este estudo objetivou apresentar a operacionalização do MARP em serviços de saúde do estado de Santa Catarina, de modo a subsidiar reflexões acerca da aplicabilidade desse método no controle sanitário realizado pela Visa.

\section{MÉTODO}

Trata-se de um estudo descritivo de casos múltiplos holísticos. Um estudo de caso é uma investigação empírica que analisa um fenômeno contemporâneo dentro do seu contexto da vida real, especialmente quando os limites entre o fenômeno e o contexto não estão claramente definidos, tendo lugar de destaque na pesquisa de avaliação. Inclui tanto estudos de caso único (uma unidade sob avaliação), quanto de casos múltiplos (várias unidades sob avaliação). São classificados como holísticos, se possuem apenas uma unidade de análise ${ }^{13}$.

A unidade de análise deste estudo é o controle sanitário de serviços de saúde realizado pela Visa estadual de SC em serviços de saúde desse estado, chamados nesta metodologia de "casos".

A Visa de SC iniciou em 2017 a utilização do MARP \& ROI nos hospitais da Grande Florianópolis, que constitui uma região com 22 municípios. Entre os 35 hospitais localizados nessa região, 15 (42,9\%) participaram do estudo, que representaram os "casos" desse estudo, por possuírem UTI, CC e CME.

A estratégia de avaliação de risco adotada para esses serviços de saúde foi o MARP com aplicação dos ROI, no qual os indicadores de controle de riscos presentes nas legislações sanitárias são contemplados, classificados em crítico ou não crítico e descritos em seis possíveis situações ( 0 a 5 ).

Para cada serviço/unidade da organização hospitalar estudada, foi elaborado um ROI, utilizado como instrumento legal para inspeção, e as normas sanitárias consideradas padrão-ouro de avaliação do MARP.

Cada ROI foi disponibilizado em arquivo formato pdf e, após aplicação, os dados foram inseridos no Sistema de Informação Estadual de Risco e Benefício Potencial (SIERBP), implantado pelo estado de SC, através da Resolução Normativa $n^{\circ} 3$, de 27 de setembro de $2019^{14}$. Esse sistema objetiva obter registros informatizados sobre os serviços de saúde e de interesse da saúde, bem como gerenciar e padronizar as inspeções e autoinspeções sanitárias usando a metodologia MARP e o instrumento de avaliação ROI.

Neste estudo, estamos apresentando os dados do controle sanitário das unidades CC, CME e UTI, avaliadas segundo seus ROI, específicos e aplicados nos hospitais estudados pelos profissionais da Visa de SC, de forma pontual nos anos 2017, 2018 e 2019.

\section{RESULTADOS E DISCUSSÃO}

Apresentamos a seguir os resultados da aplicação dos ROI segundo MARP, das unidades CC, CME e UTI dos hospitais inspecionados pela Visa de SC, no período compreendido entre os anos de 2017 a 2019. 


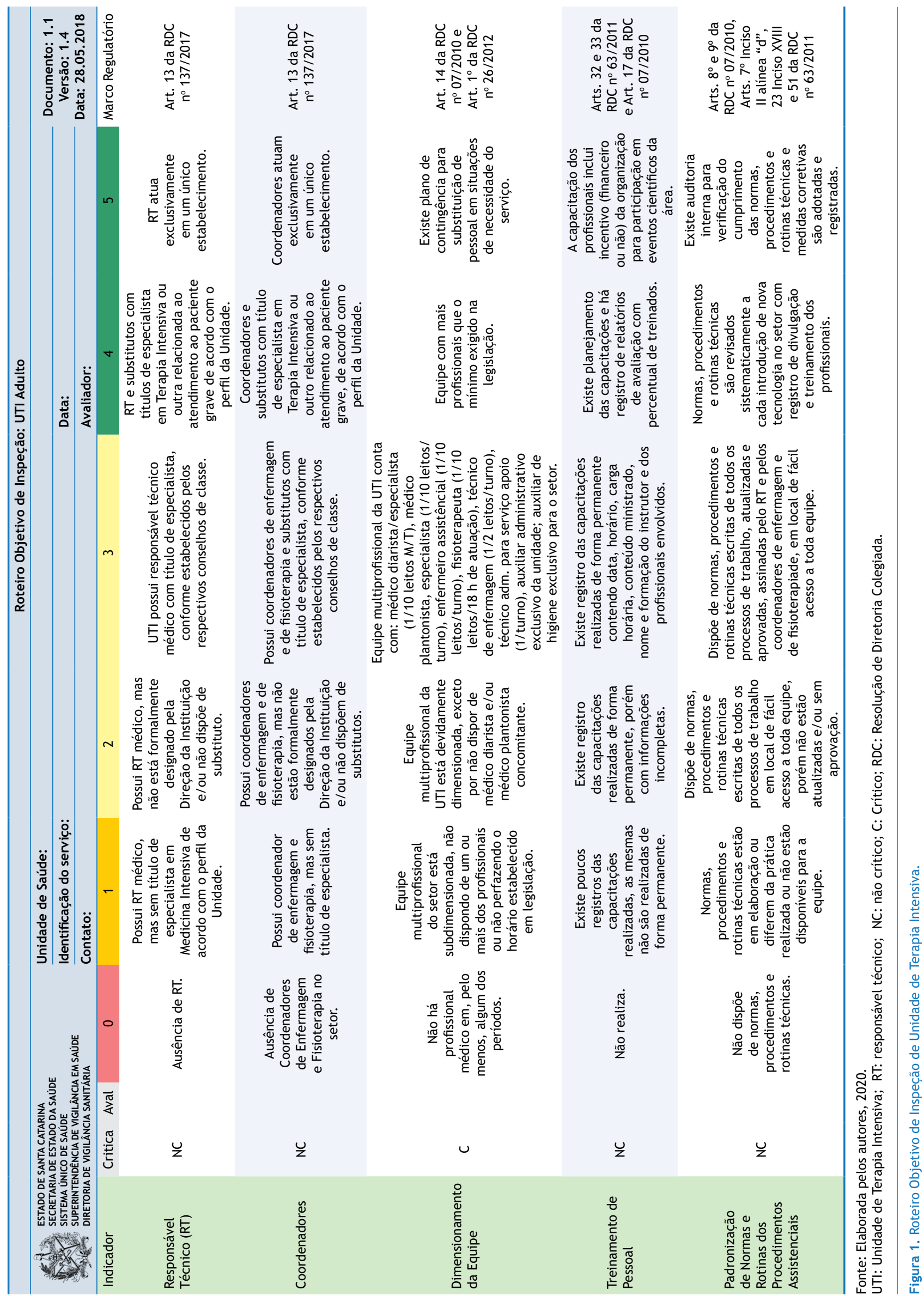


Quadro. Classificação de risco potencial (RP).

\begin{tabular}{|lc|}
\hline Classificação dos valores de RP & \\
\hline Aceitável & $\mathrm{RP} \leq 0,049$ \\
Tolerável & $0,049<\mathrm{RP} \leq 0,360$ \\
Inaceitável & $\mathrm{RP}>0,360$ \\
\hline
\end{tabular}

Fonte: Elaborado pelos autores, 2020.

A Figura 2 apresenta a avaliação de risco potencial classificado nessa metodologia como aceitável, tolerável e não aceitável dos hospitais localizados na Grande Florianópolis.

Observa-se na Figura 2 que as três unidades avaliadas possuem os três níveis de risco previstos pelo MARP, sendo que as UTI apresentam os maiores percentuais de risco aceitável (52\%), seguido dos CC (42\%) e por último das CME (31\%). A classificação de risco tolerável dessas unidades foi de $36 \%$ nas UTI, $37 \%$ nos CC e $37 \%$ nas CME. 0 risco classificado como não aceitável foi maior nas CME (31\%) seguido dos CC (21\%) e das UTI (12\%).

Esses dados revelam que, das três unidades avaliadas nos hospitais da Grande Florianópolis, as UTI possuem os maiores percentuais de risco aceitável e os menores de risco não aceitável e as CME possuem os maiores percentuais de risco não aceitável e os menores de risco aceitável.

As Figuras 3, 4 e 5 apresentam a classificação de risco dos hospitais da Grande Florianópolis segundo o número de leitos, utilizando a classificação adotada por De Negri ${ }^{15}$, na qual hospitais de pequeno porte são os que possuem até 50 leitos, os de médio porte de 51 a 150 leitos e hospitais de grande porte são os que possuem mais de 150 leitos.

Na Figura 3, observa-se que os CC dos hospitais de grande porte da grande Florianópolis, apresentam os maiores percentuais de risco aceitável (75\%), seguidos pelas UTI (45\%) e CME (25\%). 0 risco tolerável nas três unidades avaliadas foi identificado em $75 \%$ das CME, 33\% nas UTI e ausente nos CC. O risco não aceitável é maior nos CC (25\%), seguido nas UTI (22\%) e nenhum percentual desse risco nas CME desses hospitais.

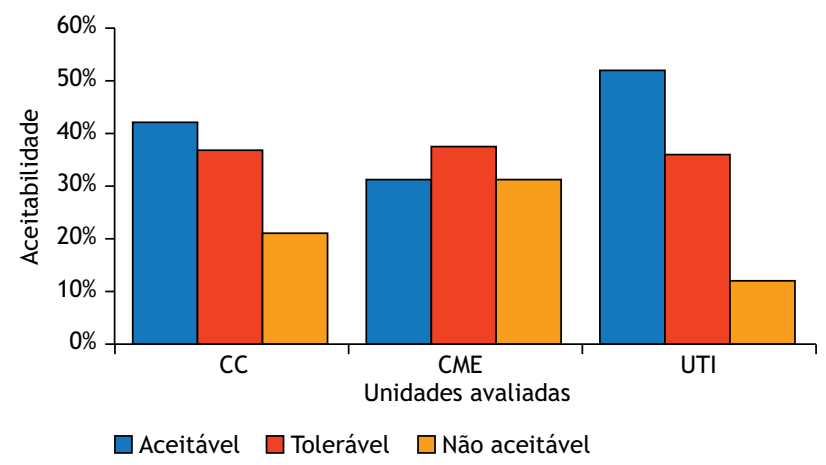

Fonte: Elaborada pelos autores, 2020.

CC: Centro Cirúrgico; CME: Central de Material e Esterilização; UTI: Unidade de Terapia Intensiva.

Figura 2. Classificação de risco segundo o Modelo de Avaliação de Riscos Potenciais (MARP) aplicado em hospitais localizados na Grande Florianópolis no período de 2017 a 2019.
Esses dados apontam que o risco potencial aceitável é maior nos CC dos hospitais de grande porte, mas também essas unidades têm os maiores percentuais de risco não aceitável. As CME apresentam os menores percentuais de risco aceitável e nenhum risco não aceitável e as UTI dos hospitais de grande porte, risco potencial nas três classificações, sendo o aceitável de $45 \%$.

Nos hospitais de médio porte, o risco potencial aceitável tem uma distribuição mais uniforme, com percentuais de $73 \%$ nas UTI, $57 \%$ nas CME e $50 \%$ nos CC. O risco tolerável é maior nos CC (40\%), seguidos das UTI (27\%) e das CME (14\%). O risco não aceitável é ausente nas UTI, mas presente nas CME (29\%) e nos CC (10\%).

Os dados da avaliação de risco dos hospitais de médio porte, destoam dos dados identificados nos hospitais de grande porte, ao revelar que as unidades de maior aceitabilidade de risco são as UTI (73\%), enquanto nos hospitais de grande porte são os CC. As UTI são também as unidades onde o risco inaceitável está ausente.

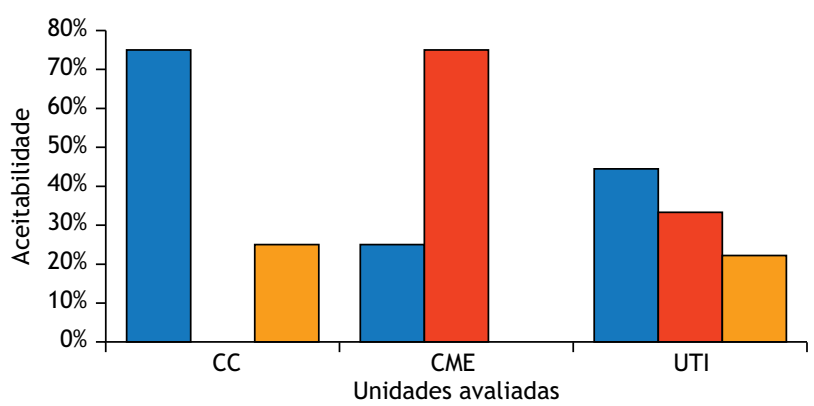

$\square$ Aceitável $\square$ Tolerável $\square$ Não aceitável

Fonte: Elaborada pelos autores, 2020.

CC: Centro Cirúrgico; CME: Central de Material e Esterilização; UTI: Unidade de Terapia Intensiva.

Figura 3. Classificação de risco segundo Modelo de Avaliação de Riscos Potenciais (MARP) em hospitais de grande porte localizados na Grande Florianópolis no período de 2017 a 2019.

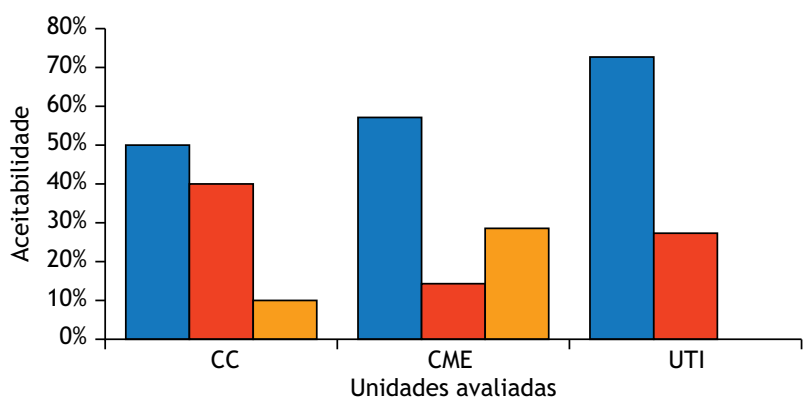

$\square$ Aceitável $\square$ Tolerável $\square$ Não aceitável

Fonte: Elaborada pelos autores, 2020.

CC: Centro Cirúrgico; CME: Central de Material e Esterilização; UTI: Unidade de Terapia Intensiva.

Figura 4. Classificação de Risco segundo Modelo de Avaliação de Riscos Potenciais (MARP) em hospitais de médio porte localizados na Grande Florianópolis no período de 2017 a 2019. 
A Figura 5 apresenta um predomínio do risco potencial classificado como tolerável e não aceitável nos hospitais de pequeno porte da Grande Florianópolis. Os percentuais de risco tolerável são de $60 \%$ nos CC e UTI e de $40 \%$ nas CME e os de risco não aceitável é de $60 \%$ nas CME, de $40 \%$ nos CC e de $20 \%$ nas UTI. O risco aceitável é ausente nos $\mathrm{CC}$ e nas $\mathrm{CME}$ desses hospitais.

Os resultados da avaliação de risco, segundo o MARP, oriundos da ausência ou não atendimento às normas sanitárias expedidas pela Agência Nacional de Vigilância Sanitária (Anvisa) e aplicados nos hospitais desse estudo, revelaram que este método é exequível e efetivo na identificação de riscos potenciais desses serviços ${ }^{12}$.

As unidades CC, UTI e CME para essa avaliação são setores considerados de alta especificidade assistencial, pilares para a qualidade do cuidado hospitalar e essenciais para a segurança do paciente e prevenção de eventos adversos relacionados às cirurgias, aos procedimentos invasivos e ao reúso e reprocessamento de dispositivos médicos ${ }^{16}$.

Identificou-se que, nos hospitais da Grande Florianópolis, as UTI apresentaram os maiores percentuais de risco aceitável e os menores de risco não aceitável, enquanto, inversamente, as CME revelaram os menores percentuais de risco aceitável e os maiores de risco não aceitável. Os CC apresentaram $42 \%$ de risco aceitável e $21 \%$ de risco não aceitável, indicando, nesse estudo, que as UTI se destacam como as unidades com melhores desempenhos de avaliação de risco potencial, seguidos pelos CC e pelas CME.

Essa avaliação constitui o primeiro estudo quantitativo de risco potencial em serviços hospitalares no Brasil e, a despeito da incipiência do uso dessa metodologia aplicada ao controle sanitário de serviços de saúde da Visa, esses dados apontam a necessidade de melhoria dos processos organoestruturais e assistenciais das três unidades avaliadas, especialmente para as CME.

O percentual de risco aceitável de $52 \%$ para unidades de tratamento crítico, que atendem majoritariamente pacientes graves

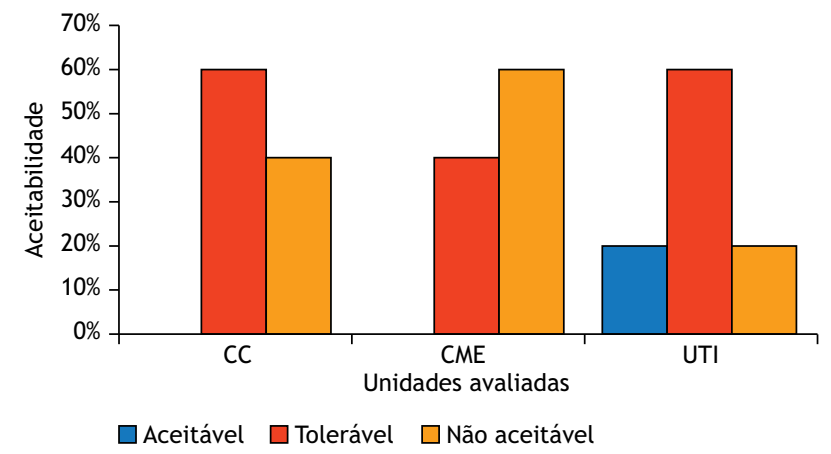

Fonte: Elaborada pelos autores, 2020.

CC: Centro Cirúrgico; CME: Central de Material e Esterilização; UTI: Unidade de Terapia Intensiva.

Figura 5. Classificação de Risco segundo Modelo de Avaliação de Riscos Potenciais (MARP) em hospitais de pequeno porte localizados na Grande Florianópolis no período de 2017 a 2019. e que requerem procedimentos invasivos e uso maciço de drogas, incluindo antimicrobianos, nos parece um percentual aquém do desejado, mesmo tendo em vista que nesse momento, não existem percentuais de risco aceitável para unidades críticas que possa servir como benchmark ou indicador padrão para efeitos de melhor análise.

Mesma racionalidade para os CC que constituem unidades de grande complexidade pelos procedimentos de risco realizados e para as CME setores responsáveis pelos processos de limpeza, desinfecção, esterilização e controles de qualidade dos produtos médicos utilizados na maioria dos procedimentos assistenciais e que apresentaram, neste estudo, baixos percentuais de risco aceitável, como $42 \%$ e $31 \%$, respectivamente.

Nesse sentido, é importante ratificar que o controle dos riscos assistenciais estão intrinsicamente relacionados ao monitoramento da qualidade e da segurança em saúde e requerem implementação de práticas de alta confiabilidade na prestação dos cuidados de saúde, estratégia fundamental para a redução de danos ${ }^{17}$.

Adicionalmente, a qualidade e a segurança dos serviços hospitalares configuram-se como um imperativo técnico e social que devem permanecer na vanguarda do cuidado à saúde, a fim de otimizar os efeitos desejáveis (benefícios) e minimizar os indesejáveis (danos), de modo a corresponder com os patamares crescentes de exigência dos usuários por serviços de saúde seguros e de risco controlado, o que demanda do Estado, particularmente das Visa, efetividade das ações, tomada de decisões relevantes e reorientação das suas estratégias de controle sanitário ${ }^{18,7,10}$.

A análise estratificada do risco potencial dos hospitais estudados, segundo o número de leitos versus unidades avaliadas, traz um panorama distinto do apresentado na Figura 2.

Os percentuais de risco aceitável são maiores nos CC (75\%) dos hospitais de grande porte, maiores nas UTI (73\%) dos hospitais de médio porte e ausente nas CME e CC dos hospitais de pequeno porte. Esses dados podem refletir diversas situações, a exemplo de maiores investimentos em recursos humanos e tecnológicos nos hospitais com grandes demandas assistenciais (grande e médio porte). Podem também refletir maior controle sanitário por parte da Visa, uma vez que são organizações consideradas mais complexas e com maior visibilidade social.

Nos hospitais de médio porte, predominam os maiores percentuais de risco aceitável desse estudo, com taxas de 73\% nas UTI, $57 \%$ nas CME e $50 \%$ nos CC. Os percentuais de risco tolerável e inaceitável são os mais prevalentes nos hospitais de pequeno porte, em todas as três unidades avaliadas, confirmando os dados anteriores e destacando a necessidade do reforço do controle sanitário nesses serviços.

A aplicabilidade do MARP pela Visa de SC nos hospitais da Grande Florianópolis possibilitou a identificação dos riscos potenciais em unidades de grande relevância, instrumentalizando o órgão sanitário para a atuação pautada em indicadores segundo as normas sanitárias vigentes, tendo os resultados 
apontados a concordância com autores que advogam o uso de um arcabouço metodológico quantitativo de risco e benefício, na avaliação de estruturas complexas, como os serviços hospitalares aqui estudados ${ }^{5}$.

Os resultados quantitativos e parametrizados desse método permitiram a classificação da aceitabilidade dos riscos potenciais, tanto das unidades avaliadas (UTI, CME e CC) quanto por porte do hospital. Com isso a Visa identificou quais os principais problemas e onde estão, de modo a direcionar suas ações para as unidades e hospitais de maior risco potencial.

Esses resultados também permitem o acompanhamento da evolução temporal dos riscos potenciais dos serviços, proporcionando a construção das suas historicidades, indicando tendências de conformidades ou não conformidades. Adicionalmente, subsidia a Visa na tomada efetiva de ações de prevenção e controle de riscos, na medida em que, ao analisar uma tendência histórica, poderá intervir no serviço, antes da mudança da classificação de risco, como de aceitável para tolerável ou até mesmo de aceitável para inaceitável.

Importante ressaltar a reprodutibilidade e transparência que o método agrega ao processo de inspeção sanitária, já que identifica objetivamente a situação de cada indicador de controle de risco, tornando possível que o setor regulado saiba inequivocamente qual a situação identificada, bem como reduz significativamente a pessoalidade do processo de avaliação, evitando que diferentes avaliadores indiquem diferentes situações encontradas.

Além de ser útil nas mais diversas práxis da Visa, que vão desde a regulação até a inspeção, o MARP \& ROI pode ser uma ferramenta também a ser utilizada no processo de contratualização das secretarias de saúde, de modo a contribuir para a melhoria da qualidade e da segurança dos serviços ofertados pelo SUS, a exemplo da política hospitalar catarinense de 2019, que incluiu o MARP \& ROI como um dos critérios para contratualização ${ }^{14}$.

\section{CONCLUSÕES}

Este estudo é pioneiro ao apresentar dados de inspeção sanitária em serviços hospitalares, utilizando uma metodologia centrada no risco potencial, principiando, assim, um caminho distinto do até então trilhado pelos serviços de Visa brasileiros e um novo paradigma de controle sanitário.

A avaliação de risco e benefício em serviços hospitalares usando um modelo quantitativo favorece o gerenciamento dos riscos em vigilância sanitária e o direcionamento de suas ações de controle sanitário. Entretanto, tem limitações, ao necessitar de um sistema de informação e apresentar dificuldades operacionais para alguns serviços. Outra consideração diz respeito à compreensão dos resultados dos dados, tendo em vista que risco e benefício são conceitos desafiadores e devem ser analisados num contexto definido.

\section{REFERÊNCIAS}

1. Silva JAA, Costa EA, Luchese G. SUS 30 anos: vigilância sanitária. Cienc Saude Coletiva. 2018;23(6):1953-62. https://doi.org/10.1590/1413-81232018236.04972018

2. Almeida N, Rouquayrol MZ. Epidemiologia \& saúde. 8a ed. Rio de Janeiro: Medsi; 2008.

3. Navarro MVT. Risco, radiodiagnóstico e vigilância sanitária. Salvador: Universidade Federal da Bahia; 2009.

4. Macedo EV, Delgado IF, Gemal AL. Método para avaliação do risco potencial no âmbito dos laboratórios oficiais: método ARP-LAB. Vigil Sanit Debate. 2015;3(3):4-10. https://doi.org/10.3395/2317-269x.00708

5. Bellanti F, Van Wijk RC, Danhof M, Pasqua OD. Integration of PKPD relationships into benefit-risk analysis. Br J Clin Pharmacol. 2015;80(5):979-91. https://doi.org/10.1111/bcp.12674

6. Freitas VLSM, Leandro KC, Navarro MVT. 0 olhar do benefício além do risco: construindo um novo paradigma em vigilância sanitária. Rev Bras Fis Medica. 2019;13(1):128-37. https://doi.org/10.29384/rbfm.2019.v13.n1.p128-137

7. European Medicines Agency - EMA. Benefit-risk methodology project: work package 2 report: applicability of current tools and processes for regulatory benefit-risk assessment. London: European Medicines Agency; 2011.
8. Warren JB, Day S, Feldschreiber P. Symmetrical analysis of risk-benefit. Br J Clin Pharmacol. 2012;74(5):757-61. https://doi.org/10.1111/j.1365-2125.2012.04265.x

9. Jiang Q, He W. Benefit-risk: assessment methods in medical product development. London: Chapman \& Hall; 2016.

10. Mussen F. Benefit-risk assessment. In: Marsh K, Goetghebenr M, Thokala P, Baltussen R, editors. Multi-criteria decision analysis to support healthcare decisions. Cham: Springer; 2017. p. $105-18$

11. Costa EAM. Vigilância sanitária de serviços de saúde: os desafios da prática. Vigil Sanit Debate. 2014;2(2):27-33. https://doi.org/10.3395/vd.v2i2148

12. Navarro MVT. Conceito e controle de riscos à saúde em radiodiagnóstico: uma abordagem de vigilância sanitária [doutorado]. Salvador: Universidade Federal da Bahia; 2007.

13. Yin RK. Estudo de caso: planejamento e métodos. 3a ed. Porto Alegre: Bookman; 2005.

14. Secretaria Estadual de Saúde de Santa Catarina. Resolução Normativa $\mathrm{N}^{\circ} 3$, de 27 de setembro de 2019. Implanta o sistema de informação estadual de risco e benefício potencial Sierbp, com objetivo de obter registros informatizados sobre os serviços de saúde e de interesse da saúde, bem como gerenciar e padronizar as inspeções e autoinspeções sanitárias. Diário Oficial do Estado. 30 set 2019. 
15. Negri A. O papel dos hospitais na rede de atenção à saúde. Consensus. 2014;4(11):12-23.

16. Travassos C, Caldas B. A qualidade do cuidado e a segurança do paciente: histórico e conceito. In: Agência Nacional de Vigilância Sanitária - Anvisa. Assistência segura: uma reflexão teórica aplicada à prática. Brasília: Agência Nacional de Vigilância Sanitária; 2017. p. 19-28.
17. Thull-Freedman J, Mondoux S, Stang A, Chartier LB. Going to the COVID-19 Gemba: using observation and high reliability strategies to achieve safety in a time of crisis. CJEM. 2020;22(6):738-41. https://doi.org/10.1017/cem.2020.380

18. Maia CS, Freitas DRC, Guilherme D, Azevedo AF. Percepções sobre qualidade de serviços que atendem à saúde da mulher. Cienc Saude Coletiva. 2011;16(5):2567-74. https://doi.org/10.1590/S1413-81232011000500027

\section{Contribuição dos Autores}

Navarro MVT, Costa EAM, Freitas VLSM, Duarte LGC - Concepção, planejamento (desenho do estudo), aquisição, análise, interpretação dos dados e redação do trabalho. Freitas L, Kindermann C - Aquisição, análise, interpretação dos dados e redação do trabalho. Todos os autores aprovaram a versão final do trabalho.

Conflito de Interesse

Os autores informam não haver qualquer potencial conflito de interesse com pares e instituições, políticos ou financeiros deste estudo.

Licença CC BY-NC atribuição não comercial. Com essa licença é permitido acessar, baixar (download), copiar, imprimir, compartilhar, reutilizar e distribuir os artigos, desde que para uso não comercial e com a citação da fonte, conferindo os devidos créditos de autoria e menção à Visa em Debate. Nesses casos, nenhuma permissão é necessária por parte dos autores ou dos editores. 